\title{
A rare case of isolated schwannoma in infraorbital nerve
}

June Seok Choi ${ }^{1}$,

Sang Min Park ${ }^{1}$,

Kyung-Un Choi ${ }^{2}$

Departments of ${ }^{1}$ Plastic and

Reconstructive Surgery and ${ }^{2}$ Pathology,

Pusan National University Hospital,

Busan, Korea
A schwannoma is a benign tumor that develops from Schwann cells. It is known to occur more frequently in women than men, and about one third of schwannoma cases occur in the head and neck area. It is also known to originate mainly in the auditory nerve. However, it is rarely associated with the trigeminal nerve, and especially, schwannomas related to the infraorbital nerve are very rare. we report a rare case of a schwannoma involving the infraorbital branch of the trigeminal nerve in a 45-year old male adult. The patient underwent physical examination and magnetic resonance imaging. The mass was approached through subciliary approach that is familiar to the plastic surgeon and completely resected. Histopathological findings showed pointed to a benign schwannoma. Infraorbital nerve schwannoma is difficult to distinguish from other diseases by means of clinical symptoms, physical findings, or imaging. In spite of its rarity, infraorbital nerve schwannoma may be considered a possible diagnosis in the case of mass on cheek. Assessment by computed tomography or magnetic resonance imaging is necessary for proper diagnosis. About the surgical approach, excision through the subciliary approach should be considered rather than the direct transfacial approach in view of stability, cosmetic effects, and familiarity.

Keywords: Schwann cells / Cheek / Surgical procedures

\section{INTRODUCTION}

A schwannoma is a benign tumor that develops from Schwann cells. It is known to occur more frequently in women than men, and it develops mostly in upper arms, wrists, and knees, but about one third of schwannoma cases occur in the head and neck area. It is also known to originate mainly in the auditory nerve. However, it is rarely associated with the trigeminal nerve, and especially, schwannomas related to the infraorbital nerve are very rare [1-4]. Since it is resistant to radiation, the treatment of choice is complete surgical excision in principle, usually using a direct transfacial approach [1,5]. The authors experienced a rare case of schwannoma arising from the infraorbital

\section{Correspondence: Sang Min Park}

Department of Plastic and Reconstructive Surgery, Pusan National University

Hospital, 179 Gudeok-ro, Seo-gu, Busan 49241, Korea

E-mail: sobbap@naver.com

Received July 13, 2018 / Revised August 31, 2018 / Accepted September 12, 2018 nerve in the right cheek of a 45-year-old male patient and performed surgery through the subciliary approach, so we report this case with a review of the literature.

\section{CASE REPORT}

A 45-year-old male patient presented with the chief complaint of the palpable mass in the right cheek area which was found 3-4 years before he came to the hospital. The mass was found to be solid on palpation, and the patient did not complain of other symptoms such as tenderness and did not have any trauma history (Fig. 1). On facial magnetic resonance imaging, a relatively well-circumscribed mass sized $1.7 \times 2.1 \times 2.3 \mathrm{~cm}$ was observed in the right cheek area. The image showed peripheral enhancement of the mass, and the inside included a necrotic portion showing low signal intensity (SI) on T1 weighted image and high SI on T2 weighted image (Fig. 2). 


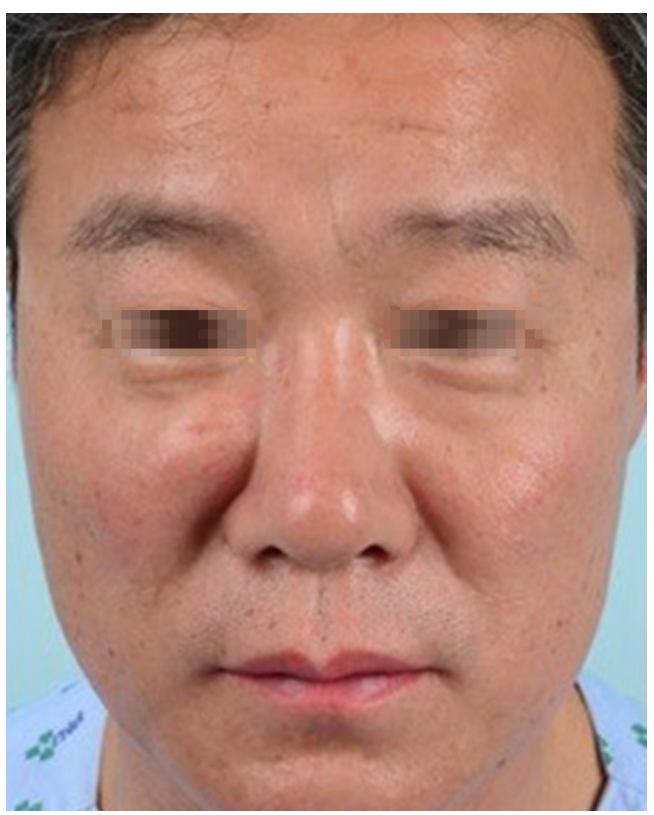

Fig. 1. A 45-year-old male patient presented with the chief complaint of the palpable mass in the right cheek area.
In addition, involvement of adjacent orbicularis oculi muscles was observed, and there were restrictions on the evaluation due to the air of the right maxillary sinus, but the findings suggestive of involvement of adjacent maxilla were not definite. Based on physical examination and imaging findings, we planned to perform resection through a subciliary approach, with a higher possibility of a true mass than vascular malformation in mind.

Under general anesthesia, the orbital floor and zygoma were exposed through the subciliary incision, the tumor was carefully dissected, and the mass was completely excised paying heed to the margins along the boundary with surround tissues. No evidence of the invasion of the tumor tissue into neighboring tissues was observed. We were careful to spare nerve from excision and used small blunt scissors and bipolar electrocautery. We opened epineurium and separated mass from the nerve fascicles.

Intraoperative frozen section biopsy results were suggestive of schwannoma. On final histologic examination, the tissue specimen was positive for s-100 protein immunohistochemical
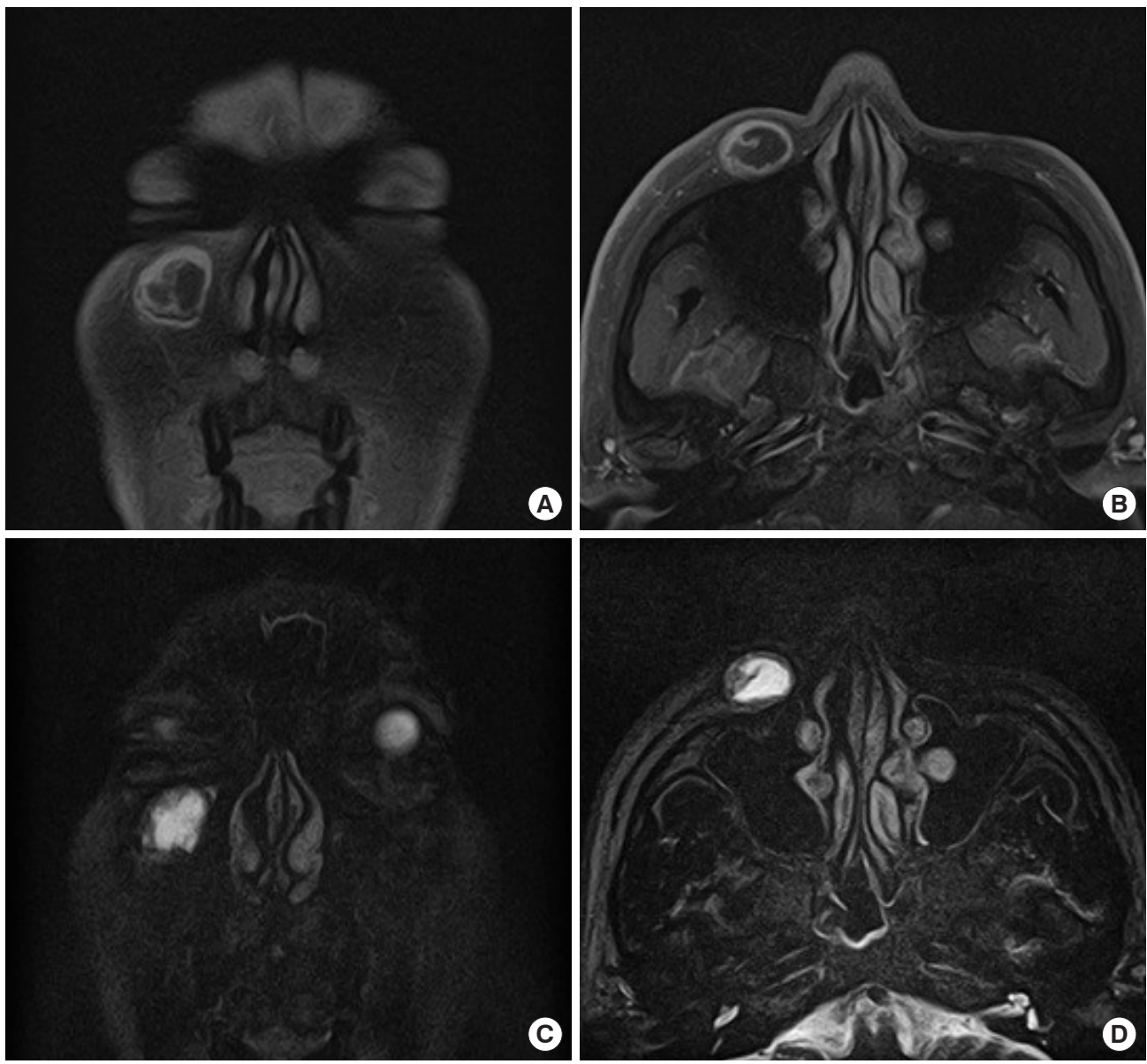

Fig. 2. (A) Relatively well-circumscribed mass sized $1.7 \times 2.1 \times 2.3 \mathrm{~cm}$ was observed in the right cheek area. The image showed peripheral enhancement of the mass, and the inside included a necrotic portion showing low signal intensity (SI) on T1 weighted image and high SI on T2 weighted image. (B) Preoperative axial magnetic resonance imaging (MRI, T1). (C) Preoperative coronal MRI (T2). (D) Preoperative axial MRI (T2). 

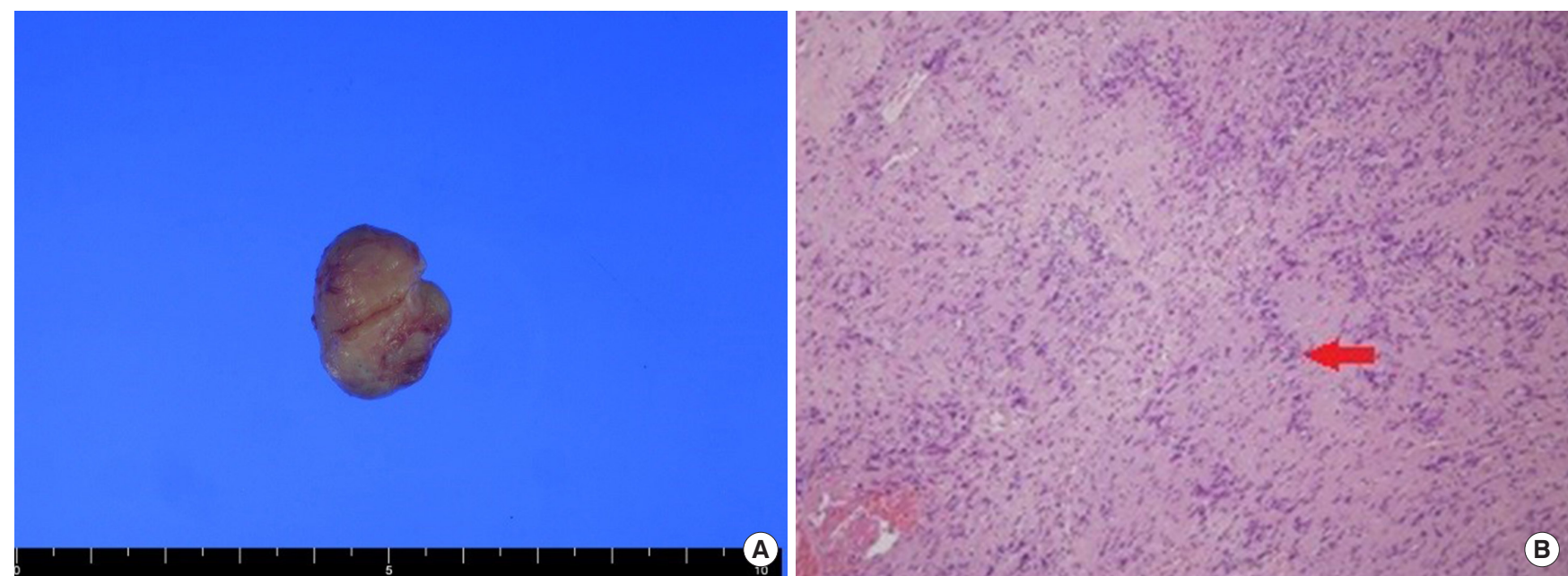

Fig. 3. (A) Gross finding: $1.7 \times 2.0 \times 1.6 \mathrm{~cm}$, the tumor was encapsulated within a fibrous pseudocapsule. (B) Photomicrograph of a tumor specimen showing a biphasic pattern of Antoni A area (arrow) with Verocay bodies (H\&E, $\times 40)$.

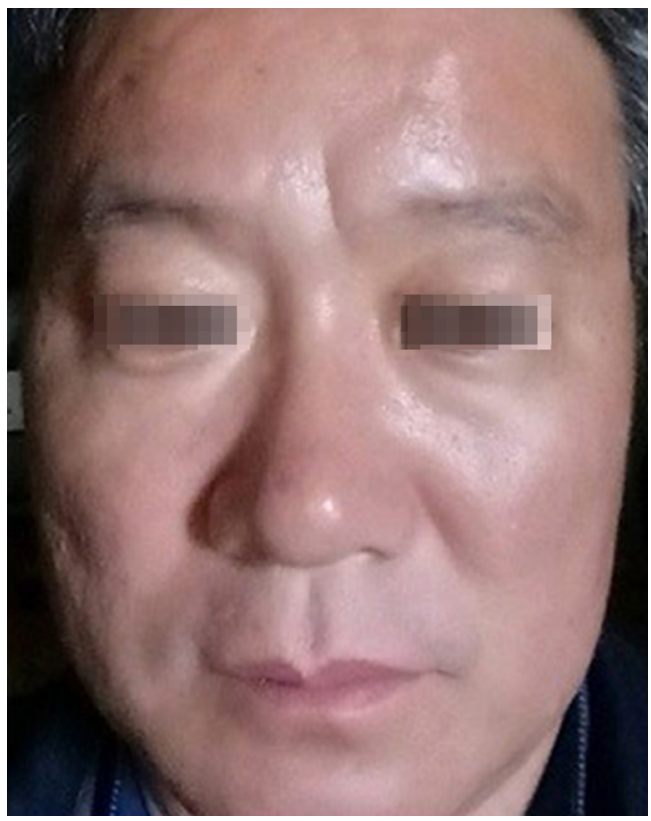

Fig. 4. Postoperative photograph of the patient after 2 years.

staining, confirming the diagnosis of schwannoma (Fig. 3). The patient has been followed up for 2 years after surgery and no other symptoms such as sensory abnormality are reported without any findings of recurrence (Fig. 4).

\section{DISCUSSION}

A schwannoma is a benign tumor that arises from Schwann cells. It can occur in any body part with a sheathing myelinated nerve. There is no predilection for any particular sex or race. It can occur in all ages. Most commonly found in individuals between 20 and 70 years of age [4]. About a third of the cases oc- cur in the head and neck area, and it is known to originate primarily in the auditory nerve. However, it is rarely related to the trigeminal nerve, and especially, it is very rarely associated with the infraorbital nerve. Only 11 cases have been reported in literature [1-3].

Diagnosis is generally made by the patient's medical history, symptoms, and imaging tests, and can be confirmed by histopathologic examination through special immunohistochemical staining. Histologically, a schwannoma is composed of Schwann cells, and has no fibroblasts, mast cells, neurilemmal cells, or endoneurial cells. It is easily diagnosed if cytoplasmic processes called Verocay bodies are found [6]. Clinical symptoms vary depending on the distribution of the mass and the origin of the dominant nerve, but patients mostly complain of compression, pain or sensory disturbance due to the mass. In patients, there were no specific symptoms other than the palpable mass in the right cheek area and discomfort when wearing glasses.

Physical examination revealed that it was a nonspecific cystic mass, and it was difficult to distinguish it from other tumors occurring in the head and neck region. The imaging findings are characterized by partial high density enhancement within the mass distinct from the surrounding cystic structure on the computed tomography scan. Magnetic resonance imaging is characterized by moderate SI on T1 and various signal intensities on T2. In this case, since low SI on T1 and high SI on T2 were observed, it was difficult to suspect it to be a case of schwannoma, and histological resection and examination were necessary for confirmation.

In principle, a schwannoma should be removed by surgical excision because of its resistance to radiation therapy, and in other literature, the transfacial approach has been introduced as 
an approach to trigeminal schwannomas [7]. In this case, the tumor was somewhat distant from the location where it was palpable, and there were concerns about the invasion into surrounding tissues or difficulty of dissection. However, considering several factors such as safety problems like facial nerve damage, cosmetic problems such as scar formation, and familiar plastic surgical procedures, we approached it through subciliary incision, and since the tumor did not invade the neighboring tissue and was well dissected, it was possible to excise it sufficiently.

In this case, among the head and neck tumors, a rare case of schwannoma occurring in the cheek area was introduced, and it was completely excised through the subciliary approach. This case shows that the infraorbital nerve schwannoma needs to be considered in the diagnosis of a mass in the cheek area. In addition, since an infraorbital nerve schwannoma is difficult to distinguish from other diseases by means of clinical symptoms, physical findings, or imaging tests, extra care is required in making a diagnosis. Finally, in relation to the surgical approach, excision through the subciliary approach should be considered rather than the direct transfacial approach in view of stability, cosmetic effects, and familiar plastic surgical procedures.

\section{CONFLICT OF INTEREST}

No potential conflict of interest relevant to this article was reported.

\section{PATIENT CONSENT}

The patients provided written informed consent for the publication and the use of their images.

\section{REFERENCES}

1. Champagne PO, Desrosiers M, Moumdjian R. Endoscopic resection of an infraorbital nerve schwannoma. Clin Neurol Neurosurg 2014;119:106-9.

2. Kok YO, Yeo MS, Nallathamby V, Lee SJ. Infraorbital nerve schwannoma presenting as an upper lip mass in an adolescent boy. Ann Plast Surg 2013;71:196-7.

3. Naina P, Masih D, Mathews SS. Infraorbital nerve schwannoma: a rare cause of upper jaw swelling. Indian J Dent Res 2014; 25:263-5.

4. Garg R, Dhawan A, Gupta N, D’souza P. A rare case of benign isolated schwannoma in the inferior orbit. Indian J Ophthalmol 2008;56:514-5.

5. Kumar N. Infra-orbital nerve schwannoma: report and review. J Nat Sci Biol Med 2015;6:267-71.

6. Kim KS, Jung JW, Yoon KC, Kwon YJ, Hwang JH, Lee SY. Schwannoma of the orbit. Arch Craniofac Surg 2015;16:67-72.

7. Karkas AA, Schmerber SA, Bettega GV, Reyt EP, Righini CA. Osteoplastic maxillotomy approach for infraorbital nerve schwannoma, a case report. Head Neck 2008;30:401-4. 